\title{
Transtorno de Estresse Pós-Traumático e funções cognitivas
}

\author{
Christian Haag Kristensen ${ }^{1}$ \\ Maria Alice de Mattos Pimenta Parente \\ Alfred W. Kaszniak
}

\begin{abstract}
Resumo
O Transtorno de Estresse Pós-Traumático (TEPT) é um transtorno prevalente, associado a alterações neurobiológicas e cognitivas. O presente artigo é um estudo teórico sobre as aplicações da avaliação neuropsicológica no TEPT. Inicialmente são revisados aspectos conceituais do transtorno, bem como alterações neurobiológicas relacionadas aos prejuízos cognitivos comumente detectados. Finalmente, argumenta-se que a avaliação dos prejuízos nas funções de memória, atenção e, sobretudo, funções executivas são relevantes tanto para a intervenção clínica junto ao paciente quanto para o refinamento das teorias psicológicas do TEPT.

Palavras-chave: Transtorno de Estresse Pós-Traumático; Cognição; Trauma; Funções executivas.
\end{abstract}

\section{Post Traumatic Stress Disorder and Cognitive Functions}

\begin{abstract}
Post Traumatic Stress Disorder (PTSD) is a prevalent disorder which is associated with neurobiological and cognitive impairments. This paper is a theoretical review of the uses of neuropsychological assessment in PTSD. Conceptual aspects of this disorder are reviewed, as well as the neurobiological alterations related to cognitive impairments. Finally, it is argued that the assessment of memory, attention, and executive function impairments is as relevant to the clinical intervention as it is to the refinement of the psychological theories of PTSD.

Keywords: Post Traumatic Stress Disorder; Cognition; Trauma; Executive functions.
\end{abstract}

\section{Transtorno de Estresse Pós-Traumático}

Embora a história da humanidade tenha sido acompanhada pela presença de eventos traumáticos, naturais ou provocados pelo próprio ser humano, foi somente durante a segunda metade do século XIX e início do século XX - a partir de inovações tecnológicas nos meios de transporte, que se esboçaram teorias sobre as reações humanas após experiências traumáticas. Com a progressiva utilização de locomotivas a vapor, o transporte ferroviário de passageiros cresceu e popularizou-se. Mas à proporção que um contingente maior de pessoas desfrutava desse conforto, também acidentes se tornaram relativamente freqüentes, tanto quanto disputas jurídicas por compensação financeira (Young, 2000). Interpretações divergentes para a etiologia dos fenômenos apresentados por vítimas de acidentes ferroviários foram propostas em termos: (a) "orgânicos", mais notavelmente nos trabalhos de John Erichsen (1818-1896) e Herman Oppenheim (1858-1919) ou (b) "funcionais", conforme posições adotadas por Jean-Martin Charcot (1825-1893) e alguns de seus alunos, como Pierre Janet (1859-1947) e Sigmund Freud (1856-1839).

Posteriormente, situações de guerra ofereceram grande quantidade de casos, contribuindo não somente para o refinamento de formulações teóricas, mas também para a proposição de intervenções terapêuticas variadas (Marlowe, 2000). Embora a associação entre a experiência a um evento estressor traumático e a manifestação de um conjunto de reações características possa parecer óbvia, foi somente na década de 1980 que os profissionais de saúde mental reconheceram o estresse pós-traumático como um construto psicológico válido, bem como uma condição passível de diagnóstico e tratamento (Yehuda \& McFarlane, 1995).

O Transtorno de Estresse Pós-Traumático (TEPT) é definido no DSM-IV (American Psychiatric Association, 1994) como uma resposta sintomática envolvendo revivência (critério B), esquiva de estímulos associados ao trauma e entorpecimento da responsividade geral (critério C) e excitabilidade aumentada (critério D) a um evento estressor (critério A). É um trans-

\footnotetext{
1 Endereço para correspondência:

Universidade do Vale do Rio dos Sinos - Laboratório de Neurociências

Av. Unisinos, 950 - Caixa Postal 275 - 93022-000 - São Leopoldo-RS

Fone/Fax: (51) 3332-0326

E-mail: chkristensen@yahoo.com.br
} 
torno que acarreta sofrimento clinicamente significativo e/ou prejuízo social ou ocupacional (critério F), com presença de sintomas superior a 1 mês (critério E).

$\mathrm{Na}$ população geral, é possível estimar que em torno de $60 \%$ a $90 \%$ dos indivíduos são expostos a um evento estressor potencialmente traumático ao longo da vida (Breslau e colaboradores, 1998; Kessler, Sonnega, Bromet, Hughes \& Nelson, 1995). Embora a taxa de exposição a eventos estressores seja elevada, o percentual de indivíduos na população geral que apresenta o TEPT ao longo da vida é mais baixo, podendo ser estimado entre 8\% a 9\% (Breslau e colaboradores; Kessler e colaboradores). Ainda que a grande maioria de indivíduos expostos a situações potencialmente traumáticas não desenvolva o transtorno (Yehuda \& McFarlane, 1995), o TEPT tem sido considerado o quarto transtorno mental mais comum (Yehuda, 2002), resultando em conseqüências sociais e econômicas significativas (Ballenger e colaboradores, 2004).

O diagnóstico do TEPT é fundamentalmente clínico. No entanto, apesar do esforço empregado na definição de critérios diagnósticos válidos e confiáveis, existe considerável controvérsia sobre a adequação dos critérios atuais para o TEPT (Ballenger e colaboradores, 2004; McNally, 2003). Prejuízos no funcionamento cognitivo foram incluídos nos relatos iniciais das reações pós-traumáticas (Young, 2000) e compõem, atualmente, o núcleo central do que é caracterizado no DSM como TEPT. Tem sido sugerido que a avaliação neuropsicológica possa contribuir para elucidar os prejuízos cognitivos associados ao TEPT (Horner \& Hamner, 2002; Wolfe \& Charney, 1991), esclarecendo questões diagnósticas. Assim, o objetivo deste trabalho é revisar as possíveis aplicações da avaliação neuropsicológica no TEPT, enfatizando sua relevância para o refinamento de teorias psicológicas. Para tanto, será realizada uma breve revisão da neurobiologia do TEPT, seguida pela apresentação de uma teoria sobre o processamento cognitivo no transtorno. Logo após, alguns estudos neuropsicológicos são apresentados, com particular atenção àqueles focalizando prejuízos em funções executivas.

\section{Neurobiologia do TEPT}

Durante a década de 1930, uma contribuição fundamental na investigação dos efeitos neurobiológicos à exposição a eventos estressores foi dada por Hans Selye, que reconheceu a natureza paradoxal da resposta corporal ao estresse (McEwen, 2002): os sistemas corporais ativados que auxiliam na proteção do organismo e na restauração da homeostase, se mantidos em ativação por um período prolongado, causam prejuízos ao organismo. A resposta biológica a eventos estressores envolve a ativação dos sistemas endócrino e imunológico, bem como de circuitos neurais específicos (Bremner, 2002; Charney, 2004; Graeff, 2003; Nemeroff, 2004). Já a resposta psicológica varia amplamente, incluindo desde formas adaptativas de lidar com o estresse até depressão e outras psicopatologias (Cicchetti \& Walker, 2001), entre as quais o TEPT (McNally, 2003; Yehuda, 2002).

Considerando as limitações e objetivos do presente trabalho, é importante destacar que a resposta biológica a eventos estressores depende da ativação do complexo locus ceruleus-noradrenalina-sistema nervoso simpático (Charney, 2004) e do eixo hipotálamo-hipófise-adrenal (HHA), em especial do hipotálamo (McEwen, 2002; Yehuda, 2001). Nessa região, as células do núcleo paraventricular promovem a síntese e secreção do hormônio de liberação da corticotrofina (CRH) e neuropeptídeos como a vasopressina. Pelo sistema porta esses peptídeos passam do hipotálamo à hipófise anterior, onde o CRH estimula a síntese e a liberação do hormônio adrenocorticotrófico (ACTH) na circulação sistêmica (McEwen). Por sua vez, o ACTH circulante estimula a liberação de glicocorticóides pela supra-renal. Esses hormônios esteróides adrenais - dentre os quais destaca-se no ser humano o cortisol - desempenham um papel central na resposta a eventos estressores, produzindo lipólise, glicogenólise e o catabolismo de proteínas, mantendo altas as concentrações de substratos energéticos no sangue, além de suprir a resposta imunológica do organismo (Charmandari, Kino, Souvatzoglou \& Chrousos, 2003; McEwen; Sapolsky, 2003). Em uma situação normal, ao cessar a exposição a um estressor, o eixo HHA promove uma autolimitação do sistema mas no TEPT, como será descrito abaixo, a neurobiologia segue um outro curso (Bremner, 2002; De Bellis, 2005; Graeff, 2003; Yehuda, 2001).

A investigação neurobiológica do TEPT tem privilegiado o eixo HHA e o sistema nervoso simpático pelo papel fundamental destes na resposta ao estresse (De Bellis, 2005; Nemeroff, 2004). Fisiologicamente, o TEPT pode ser conceituado como uma resposta crônica e desadaptativa. Nesse transtorno ocorre uma hiperativação do sistema nervoso simpático, caracterizada pela produção aumentada de adrenalina (epinefrina) e noradrenalina (norepinefrina) na circulação sanguínea pelos próprios terminais simpáticos e pela medula da supra-renal (Graeff, 2003; Nemeroff, 2004). Diferentemente do que ocorre em outros transtornos, como a depressão ou mesmo a resposta imediata a um estressor, no TEPT evidencia-se um aumento na sensibilidade do eixo HHA, resultando na diminuição do cortisol plasmático e urinário (Charmandari e 
colaboradores, 2003; Yehuda, 2001). Assim, a neurobiologia do TEPT caracteriza-se pela diminuição dos níveis basais de cortisol, resultante do aumento na inibição por retroalimentação (feedback) negativa, secundário ao aumento no número e sensibilidade dos receptores glicocorticóides (Yehuda, 2001).

Estruturas encefálicas como o hipocampo, o hipotálamo, a amígdala e o córtex pré-frontal estão envolvidas nos processos de retroalimentação do eixo HHA (Sapolsky, 2003) e têm sido preferencialmente identificadas em estudos clínicos com indivíduos com TEPT (Bremner, 2002; Charney, 2004). Assim, alterações no eixo HHA e no complexo locus ceruleus-noradrenalina-sistema nervoso simpático têm sido associadas a prejuízos em diversos sistemas funcionais nos indivíduos com TEPT (Bremner, 2002; De Bellis, 2005; Elzinga \& Bremner, 2002).

No entanto, as alterações associadas ao TEPT não são limitadas à desregulação de sistemas neuroquímicos, envolvendo ainda modificações na estrutura e no funcionamento encefálicos. Estudos empregando técnicas variadas de neuroimagem, como (a) imageamento por ressonância magnética, (b) tomografia por emissão de pósitrons, (c) tomografia computadorizada por emissão de fóton único, (d) imageamento por ressonância magnética funcional, ou mesmo combinações de algumas dessas técnicas têm demonstrado prejuízos estruturais e funcionais no encéfalo relacionados aos sintomas de TEPT (Bremner, 2002; para uma revisão crítica desses estudos, ver Hull, 2002). Outras metodologias, como o exame neurológico apontam na mesma direção (Gurvits e colaboradores, 2000). Tomados em conjunto, esses estudos sugerem razoável evidência sobre o prejuízo em estruturas encefálicas e funções cognitivas relacionadas ao TEPT. As principais áreas funcionalmente prejudicadas em indivíduos que desenvolveram TEPT após um evento estressor incluem: córtex préfrontal, córtex visual de associação, hipocampo, giro cingulado, amígdala e núcleo accumbens (Hull; Horner \& Hamner, 2002). Estas são estruturas funcionalmente relevantes na performance de processos integrativos, como as funções executivas, regulação do comportamento emocional, orientação espacial, e interpretação emocional, codificação e armazenamento de informação (Mesulam, 2000).

\section{Funcionamento cognitivo e avaliação neuropsi- cológica do TEPT}

Considerando a natureza do TEPT, não é surpreendente que teorias psicológicas tenham sido propostas em grande diversidade para explicar as desordens cognitivas e emocionais características do transtorno (ver revisão em Brewin \& Holmes, 2003). Entre outras tantas, uma teoria que tem recebido crescente apoio empírico é a abordagem cognitiva proposta por Ehlers e Clark (2000). O conceito central nessa abordagem é que o TEPT torna-se persistente quando uma sensação de ameaça atual, corrente, influi no processamento cognitivo do trauma. Essa sensação de ameaça (ou perigo iminente) resulta: (a) de avaliações excessivamente negativas do trauma e/ou de suas conseqüências (e sintomas) e (b) da natureza da memória do evento traumático, caracterizada por uma pobre elaboração, pobre integração em relação à memória autobiográfica, forte memória associativa e forte pré-ativação (priming) perceptual. Essa sensação de ameaça atual, uma vez ativada, é seguida pela expressão sintomatológica do TEPT (revivência persistente do evento traumático e excitabilidade aumentada) além de outros estados emocionais. Assim, além da presença de memórias do trauma pobremente elaboradas - que facilmente são ativadas por associações situacionais - um conjunto de cognições, metacognições e comportamentos contribui para a persistência do TEPT (Ehlers \& Clark).

Apesar da grande ênfase direcionada às características específicas da memória do evento traumático bem como às desordens de sistemas múltiplos de memória (Elzinga \& Bremner, 2002; McNally, 2003), prejuízos em outras funções cognitivas têm sido freqüentemente associados ao TEPT. A investigação neuropsicológica dessa associação iniciou na década de 1980 (por exemplo, Everly \& Horton, 1989), embora estudos com prisioneiros de guerra tenham sido conduzidos já em décadas anteriores. Em um trabalho seminal, Wolfe e Charney (1991) advogaram o uso da avaliação neuropsicológica para, entre outros objetivos: (a) estimar habilidades cognitivas; (b) facilitar a compreensão da sintomatologia pós-traumática, especialmente quando o evento estressor inclui um potencial dano físico ao sistema nervoso central - por exemplo, após acidentes de trânsito; (c) auxiliar no planejamento das intervenções terapêuticas; (d) favorecer a compreensão sobre o curso temporal do transtorno. Mais de uma década após a publicação de Wolfe e Charney, os avanços nessa área de investigação são notáveis. Revisões da literatura na área (Danckwerts \& Leathem, 2003; Horner \& Hamner, 2002) foram capazes de incluir em torno de 20 estudos empíricos sobre avaliação neuropsicológica no TEPT, bem como a edição de um livro recente sobre o tema (Vasterling \& Brewin, 2005).

Ao revisarem a literatura na área, Horner e Hamner (2002) observaram que os prejuízos nas funções cognitivas, verificados através de testagem neuropsicológica, apresentaram-se de forma relativamente uniforme, independentemente da natureza do evento 
estressor. Entre as funções cognitivas mais comumente prejudicadas nos indivíduos com TEPT, ao longo dos diferentes estudos revisados, encontram-se: (a) memória, em maior grau envolvendo recuperação imediata de informações verbais e visuais e, em menor grau, recuperação não-imediata; (b) atenção, nas modalidades verbal e visual; e (c) funções executivas, incluindo provas de resolução de problemas (Horner \& Hamner). Ao menos três aspectos merecem consideração adicional. Primeiro, é importante notar como os prejuízos nas funções cognitivas acima listadas são compatíveis com as alterações funcionais ou anatômicas associadas ao TEPT identificadas em estruturas encefálicas (conforme descrito na seção anterior). Estruturas como o hipocampo, amígdala, córtex pré-frontal e giro cingulado são componentes centrais do substrato neurobiológico que permite o processamento mnêmico, atencional e executivo (Mesulam, 2000; Royall e colaboradores, 2002). Segundo, esses prejuízos em funções cognitivas demonstráveis através de testagem neuropsicológica em populações clínicas apresentam-se em consonância com aqueles identificados através de estudos empíricos na tradição da psicologia cognitiva experimental, empregando tarefas de escuta dicótica, reconhecimento auditivo, procedimento de Stroop modificado (ou Stroop emocional) e memória autobiográfica, entre outras provas experimentais (McNally, 1998). Terceiro, os prejuízos em funções cognitivas demonstrados tanto através da avaliação neuropsicológica quanto da investigação experimental, não somente oferecem grande apoio empírico à abordagem cognitiva proposta por Ehlers e Clark (2000) para explicar o TEPT, como também podem ser utilizados para avançar com a teorização. Nesse sentido, além do emprego clínico para a avaliação neuropsicológica no TEPT destacado por Wolfe e Charney (1991), o presente trabalho propõe uma finalidade adicional, qual seja, sugerir a avaliação neuropsicológica como uma metodologia de investigação empírico-clínica para o avanço das teorias psicológicas do TEPT.

Como ilustração do terceiro ponto acima levantado, é possível indicar que o prejuízo em funções executivas é um componente central no modelo do processamento cognitivo no TEPT. No modelo proposto por Ehlers e Clark (2000), por exemplo, de maneira mais ou menos explícita, ênfase é dada a erros de interpretação de estímulos ambientais, dificuldades em processos de automonitoramento e prejuízos na integração de conteúdos mnêmicos. Esses são processos que envolvem justamente a participação de funções executivas (Mesulam, 2000; Royall e colaboradores, 2002), cujos prejuízos parecem ser centrais na sintomatologia do TEPT. Embora a definição de funções executivas não seja consensual, é importante notar que a investigação neuropsicológica demonstrou prejuízos em diferentes processos cognitivos associados ao TEPT dependentes do funcionamento executivo, como memória prospectiva (Moradi, Neshat-Doost, Taghavi, Yule \& Dalgleish, 1999), raciocínio abstrato (Beers \& De Bellis, 2002), flexibilidade mental (Kristensen \& Borges, 2004; Stein, Kennedy \& Twamley, 2002) e memória de trabalho (working memory, Stein e colaboradores). Em linhas gerais, o prejuízo no funcionamento executivo tem sido sugerido em estudos com indivíduos expostos a combate (Vasterling, Brailey, Constans \& Sutker, 1998), prisioneiros de guerra (Sutker, Vasterling, Brailey \& Allain, 1995) ou expostos a estressores variados, incluindo combate ou abuso sexual (Koenen e colaboradores, 2001). Adicionalmente, as estruturas encefálicas que participam dos circuitos frontosubcorticais provendo o substrato neural para $O$ desempenho das funções executivas (Heyder, Suchan \& Daum, 2004; Royall e colaboradores, 2002) têm sido implicadas no TEPT (Bremner, 2002; De Bellis, 2005; Yehuda, 2001).

\section{Considerações finais}

Embora a investigação neuropsicológica possa ser de grande utilidade no refinamento das teorias psicológicas do TEPT, algumas limitações atuais devem ser reconhecidas. Quanto aos aspectos metodológicos, é importante notar que a avaliação neuropsicológica tem sido conduzida, em boa parte dos estudos, em amostras relativamente pequenas (Horner \& Hamner, 2002). Em termos diagnósticos, a elevada comorbidade entre os participantes com TEPT em relação a outros transtornos, como Depressão Maior, Transtorno de Ansiedade Generalizada, e abuso de álcool e substâncias, dificulta a identificação do prejuízo cognitivo especificamente associado ao TEPT (Danckwerts \& Leathem, 2003; Vasterling e colaboradores, 1998). Estudos conduzidos com participantes expostos a combate ou prisioneiros de guerra são particularmente propícios a incluir, pelas características da própria população, participantes com elevada comorbidade, elevado uso de medicação, baixo nível socioeconômico e baixa escolaridade em relação à população geral (Danckwerts \& Leathem), além de problemas relacionados à motivação, como compensação financeira e benefícios por invalidez (Horner \& Hamner).

Os prejuízos no funcionamento cognitivo são aspectos constitutivos do TEPT. Não por acaso, teorias psicológicas recentes têm privilegiado desordens no processamento cognitivo de eventos estressores trau- 
máticos para explicar a ocorrência do TEPT. A avaliação neuropsicológica dos indivíduos com TEPT tem sido sugerida, entre outros fatores, por contribuir na estimativa das habilidades cognitivas, responder a questões diagnósticas diferenciais e auxiliar no planejamento terapêutico. A possibilidade de contribuições dessa natureza é suficiente para justificar a adoção da avaliação neuropsicológica como um procedimento de rotina nos pacientes com suspeita diagnóstica de TEPT.

Além das relevantes aplicações clínicas, O presente trabalho sugere que a avaliação neuropsicológica tenha um papel central no refinamento das teorias psicológicas do TEPT. Para tanto, algumas limitações metodológicas identificadas nos estudos atuais devem ser superadas. Investigações futuras na área poderiam empregar metodologias combinadas, como imageamento funcional e avaliação neuropsicológica, incluindo tarefas próximas a situações e contextos cotidianos (Danckwerts \& Leathem, 2003). Além das funções cognitivas tradicionalmente avaliadas no TEPT, como memória e atenção, tarefas e testes sensíveis a prejuízos executivos deveriam ser rotineiramente incluídos em qualquer avaliação neuropsicológica. Particularmente na avaliação das funções executivas, especial ênfase deveria ser conferida à sondagem de prejuízos cognitivos relacionados ao cotidiano dos pacientes, pois características da situação altamente estruturada na testagem neuropsicológica podem minimizar as dificuldades reais, quando a demanda pelo comportamento ocorre em um contexto flexível e errático (Mesulam, 2000), diminuindo assim a validade ecológica das tarefas propostas (Royall e colaboradores, 2002).

\section{Referências}

American Psychiatric Association. (1994). Diagnostic and statistical manual of mental disorders (4a ed.). Washington, DC: Autor.

Ballenger, J. C., Davidson, J. T. R., Lecrubier, Y., Nutt, D. J., Marshall, R. D., Nemeroff, C. B., Shalev, A. Y. \& Yehuda, R. (2004). Consensus statement update on posttraumatic stress disorder from the International Consensus Group on Depression and Anxiety. Journal of Clinical Psychiatry, 65(Supl. 1), 55-62.

Beers, S. \& De Bellis, M. D. (2002). Neuropsychological function in children with maltreatment-related posttraumatic stress disorder. American Journal of Psychiatry, 159, 483-486.
Bremner, J. D. (2002). Does stress damage the brain? Understanding trauma-related disorders from a mind-body perspective. New York: W. W. Norton.

Breslau, N., Kessler, R. C., Chilcoat, H. D., Schultz, L. R., Davis, G. C. \& Andreski, P. (1998). Trauma and posttraumatic stress disorder in the community: The 1996 Detroit Area Survey of Trauma. Archives of General Psychiatry, 55, 626-632.

Brewin, C. R. \& Holmes, E. A. (2003). Psychological theories of posttraumatic stress disorder. Clinical Psychology Review, 23, 339-376.

Charmandari, E., Kino, T., Souvatzoglou, E. \& Chrousos, G. P. (2003). Pediatric stress: Hormonal mediators and human development. Hormone Research, 59, 161-179.

Charney, D. S. (2004). Psychobiological mechanisms of resilience and vulnerability: Implications for successful adaptation to extreme stress. American Journal of Psychiatry, 161, 195-216.

Cicchetti, D. \& Walker, E. F. (2001). Stress and development: Biological and psychological consequences. Development and Psychopathology, 13, 413418.

Danckwerts, A. \& Leathem, J. (2003). Questioning the link between PTSD and cognitive dysfunction. Neuropsychology Review, 13, 221-235.

De Bellis, M. D. (2005). The psychobiology of neglect. Child Maltreatment, 10, 150-172.

Ehlers, A. \& Clark, D. M. (2000). A cognitive model of posttraumatic stress disorder. Behavior Research and Therapy, 38, 319-345.

Elzinga, B. M. \& Bremner, J. D. (2002). Are the neural substrates of memory the final common pathway in posttraumatic stress disorder (PTSD)? Journal of Affective Disorder, 70, 1-17.

Everly, G. S. \& Horton, A. M., Jr. (1989). Neuropsychology of posttraumatic stress disorder: A pilot study. Perceptual and Motor Skills, 68, 807-810.

Graeff, F. G. (2003). Bases biológicas do transtorno de estresse pós-traumático. Revista Brasileira de Psiquiatria, 25(Supl. I), 21-24.

Gurvits, T. V., Gilbertson, M. W., Lasko, N. B., Tarhan, A. S., Simeon, D., Macklin, M. L., Orr, S. P. \& Pitman, R. K. (2000). Neurologic soft signs in chronic posttraumatic stress disorder. Archives of General Psychiatry, 57, 181-186. 
Heyder, K., Suchan, B. \& Daum, I. (2004). Corticosubcortical contributions to executive control. Acta Psychologica, 115, 271-289.

Horner, M. D. \& Hamner, M. B. (2002). Neurocognitive functioning in posttraumatic stress disorder. Neuropsychology Review, 12, 15-30.

Hull, A. M. (2002). Neuroimaging findings in posttraumatic stress disorder: Systematic review. British Journal of Psychiatry, 181, 102-110.

Kessler, R. C., Sonnega, A., Bromet, E., Hughes, M. \& Nelson, C. B. (1995). Posttraumatic stress disorder in the National Comorbidity Survey. Archives of General Psychiatry, 52, 1048-1060.

Koenen, K. C., Driver, K. L., Oscar-Berman, M., Wolfe, J., Folsom, S., Huang, M. T. \& Schlesinger, L. (2001). Measures of prefrontal system dysfunction in posttraumatic stress disorder. Brain and Cognition, 45, 64-78.

Kristensen, C. H. \& Borges, J. L. (2004). Neuropsychological impairment, juvenile delinquency, and posttraumatic stress disorder: An exploratory study. Annals - 1st International Congress on Neurosciences and Rehabilitation (p. 15). Brasilia, Sarah Network of Rehabilitation Hospitals.

Marlowe, D. H. (2000). Psychological and psychosocial consequences of combat and deployment with special emphasis on the Gulf War. Santa Monica, CA: RAND.

McEwen, B. S. (com Lasley, E. N.). (2002). The end of stress as we know it. Washington, DC: DANA/Joseph Henry.

McNally, R. J. (1998). Experimental approaches to cognitive abnormality in posttraumatic stress disorder. Clinical Psychology Review, 18, 971-982.

McNally, R. J. (2003). Remembering trauma. Cambridge, MA: Belknap/Harvard.

Mesulam, M.-M. (2000). Behavioral neuroanatomy: Large-scale networks, association cortex, frontal syndromes, the limbic system, and hemispheric specializations. Em M.-M. Mesulam (Org.), Principles of behavioral and cognitive neurology (2a ed.). New York: Oxford University Press.

Moradi, A. R., Neshat-Doost, H. T., Taghavi, M. R., Yule, W. \& Dalgleish, T. (1999). Everyday memory deficits in children and adolescents with PTSD: Performance on the Rivermead Behavioural Memory Test. Journal of Child Psychology and Psychiatry, 40, 357361.
Nemeroff, C. B. (2004). Neurobiological consequences of childhood trauma. Journal of Clinical Psychiatry, 65(Supl. 1), 18-28.

Royall, D. R., Lauterbach, E. C., Cummings, J. L., Reeve, A., Rummans, T. A., Kaufer, D. I., LaFrance, W. C. \& Coffey, C. E. (2002). Executive control function: A review of its promise and challenges for clinical research. Journal of Neuropsychiatry and Clinical Neurosciences, 14, 377-405.

Sapolsky, R. M. (2003). Stress and plasticity in the limbic system. Neurochemical Research, 28, 1735-1742.

Stein, M. B., Kennedy, C. M. \& Twamley, E. W. (2002). Neuropsychological function in female victims of intimate partner violence with and without posttraumatic stress disorder. Biological Psychiatry, 52, 1079-1088.

Sutker, P. B., Vasterling, J. J., Brailey, K. \& Allain, A. N. (1995). Memory, attention, and executive deficits in POW survivors: Contributing biological and psychosocial factors. Neuropsychology, 9, 118-125.

Vasterling, J. J., Brailey, K., Constans, J. I. \& Sutker, P. B. (1998). Attention and memory dysfunction in posttraumatic stress disorder. Neuropsychology, 12, 125 133.

Vasterling, J. J. \& Brewin, C. R. (2005). Neuropsychology of PTSD: Biological, cognitive, and clinical perspectives. New York: Guilford.

Wolfe, J. \& Charney, D. S. (1991). Use of neuropsychological assessment in post-traumatic stress disorder. Psychological Assessment, 3, 573-580.

Yehuda, R. (2001). Biology of posttraumatic stress disorder. Journal of Clinical Psychiatry, 62(Supl. 17), 4146.

Yehuda, R. (2002). Current concepts: Post-traumatic stress disorder. New England Journal of Medicine, 346, 108-114.

Yehuda, R. \& McFarlane, A. C. (1995). Conflict between current knowledge about posttraumatic stress disorder and its original conceptual basis. American Journal of Psychiatry, 152, 1705-1713.

Young, A. (2000). An alternative history of traumatic stress. Em A. Y. Shalev, R. Yehuda \& A. C. McFarlane (Orgs.), International handbook of human response to trauma (pp. 51-66). New York: Kluwer/Plenum.

Recebido em abril de 2005

Reformulado em setembro de 2005 Aprovado em fevereiro de 2006 
Sobre os autores:

Christian Haag Kristensen é doutor em Psicologia do Desenvolvimento pela Universidade Federal do Rio Grande do Sul (UFRGS), bolsista PDEE-CAPES na Universidade do Arizona (2002/2003). Atualmente é professor assistente no Curso de Psicologia da Universidade do Vale do Rio dos Sinos (UNISINOS) e pesquisador no Laboratório de Neurociências (UNISINOS).

Maria Alice de Mattos Pimenta Parente é doutora em Psicologia pela Universidade de São Paulo (USP), tendo realizado Pós-Doutorado na Universidade de Montreal, Canadá e Universidade Tolouse Le-Mirail, França. Atualmente é professora titular no Instituto de Psicologia (UFRGS).

Alfred W. Kaszniak é doutor em Psicologia pela Universidade de Illinois, Chicago, EUA. Atualmente é chefe do Departamento de Psicologia na Universidade do Arizona, professor de Psicologia, Psiquiatria e Neurologia e diretor do Programa de Neuropsicologia Clínica na Universidade do Arizona. 